\title{
Optimal Controller Design for Reduced-Order Model of Rotational Mechanical System
}

\author{
Nasir Ahmed Al-awad \\ Computer Engineering Department, College of Engineering, Mustansiriyah University, Baghdad 10001, Iraq
}

Corresponding Author Email: nasir.awad@uomustansiriyah.edu.iq

https://doi.org/10.18280/mmep.070309

Received: 11 August 2020

Accepted: 9 September 2020

\section{Keywords:}

rotational mechanical system, model reduction, PID controller, LQR controller, G.A-PID

\begin{abstract}
Genetic algorithm (GA) based PID (proportional integral derivative) controller has been proposed for tuning advanced PID parameters in a Reduced-Order of Rotational Mechanical System utilizing a weighted blend of target capacities, to be specific, integral square error (ISE), integral absolute error (IAE), and integrated time absolute error (ITAE). Some classical control methods like (PID) using Ziegler-Nichols strategy, Linear-Quadratic Regulator (LQR) are also implemented for comparison. The problem here, reducing the large scale model of mechanical system and controlling by using optimal approach (GA). The results show that the GA based PID controller tuned with settled PID parameters gives acceptable execution regarding set point following when compared with classical PID and LQR.
\end{abstract}

\section{INTRODUCTION}

The displaying of the complex designing framework is a standout amongst the most vital in the control framework. In the control building field, display decrease systems are basic for the plan of controllers where specific complex techniques are included. This would furnish the originator to plan with low order controllers that have fewer equipment prerequisites and minimal effort with the same execution [1]. It is as often as possible of worry in mechanical autonomy and mechatronics regarding movement and vibration control, high exactness movement control applications on $\mathrm{CNCs}$ regularly factor responsible for higher request subordinates of position adroitly, in the event that you have mass-spring-damper (or even simply mass-spring) frameworks associated with one another in arrangement, you will wind up with higher request conditions to work with.

Bolstered bunch aging procedures are ordinarily utilized in the bioprocessing industry. A class of fourth-arrange incorporating model can be utilized to enough speak to such unpredictable elements of the fed-cluster aging procedure, numerous modern procedures and other mechanical frameworks include elements [2]. Endeavors towards getting low-arrange models from high-degree frameworks are identified with the points of determining stable lessened request models from stable unique ones and guaranteeing that the diminished request show matches comparable qualities of unique higher request system [3]. The rising general methodology for producing low-arrange models is a blended technique for the modular decrease. Initial, a general however non-ideal procedure is utilized to diminish an extensive model to a medium-sized model. At that point, a decrease strategy with an improved system is utilized to create an extremely effective low request show from the medium-sized modular. Request decrease not objective in itself it is a piece of the plan [4]. Creating models for these unpredictable frameworks can move toward becoming tedious and costly.

These procedures regularly need control frameworks so as to deliver wanted and stable outputs [5]. The issue that emerges is that the frameworks frequently contain various state factors, in this manner making them complex frameworks to show and to control. Frequently the more intricate a framework and in this manner the more unpredictable its control framework is, the more costly it is to demonstrate the procedure and also actualize the control system [6].

The rotational mechanical framework, which is connected to a drive plate, this plant was picked in this paper since it complex enough (4th arrange framework) to demonstrate that the displaying and control procedures utilized are pertinent to higher request frameworks yet it is still little enough to be effectively executed.

There are many control strategies are connected to higher request framework, yet the multifaceted nature and precariousness are shows up the principle challenge.

A low-arrange rough model of the plant is utilized for control structure with the goal that the subsequent modelbased controller has a Proportional-Integral-Derivative structure (PID). To deliver a controller with a PID structure, an assortment of techniques dependent on either efficient model decrease or controller decrease have been produced [7, 8].

Gildin [9] presents iterative Krylov subspace projection (KSP) strategies to produce low-arrange criticism controllers for brilliant wells utilizing high-arrange supply models. The principle inspiration for utilizing these techniques is to empower the proficient calculation of low-arrange store models got from the very meager structure of motions and weight coefficients after discretization.

The execution of two controllers by h-infinity and LinearQuadratic Regulator(LQR) for directing the settling chamber weight of a hypersonic wind burrow (high-arrange framework) is thought about and the plan details for h-infinity are superior to LQR technique, on the grounds that LQR needs additionally tuning and prefilter to expel the enduring state error [10].

A performance comparison between Ziegler Nichols tuned PID controller, calibrated PID controller and the proposed 
fluffy rationale controller is introduced [11]. The Fuzzy Logic controller gives no overshoot, zero enduring state blunders and littler settling time than got utilizing Ziegler Nichols tuned PID controller.

An intelligent clever methodology has been recommended to enhance the PID tuning, for example, those utilizing Genetic Algorithms (GA) [12] and the Particle Swarm Optimization (PSO) [13]. With the development of computational techniques in the ongoing occasions, improvement calculations are frequently proposed to tune the control parameters so as to locate an ideal execution [14]. The contribution of this work is studying a large scale mechanical system modelling and reduce it, using Dominant Pole Approximation method. Finally, optimal control is using (GA) to satisfied design specifications.

This paper is sorted out as, section two, the scientific model of the rotational mechanical plant, while section three, demonstrates the model request decrease, utilizing distinctive control strategies in section four, finally, the conclusion has appeared in section five.

\section{MATHEMATICAL MODEL}

The plant that will be displayed, and along these lines distinguished in this paper, is the rotational mechanical plant appeared in Figure 1. The framework is utilized to reproduce an assortment of gadget and plant forms, similar to, ServoDrive Disk System. Most broad components used to speak to physical gadgets in rotational frameworks are a snapshot of idleness, contact, switches, and riggings. This plant was picked in light of the fact that it complex enough to demonstrate that the displaying, and control methods utilized are material to higher request frameworks yet it is still little enough to be effortlessly implemented $[15,16]$.

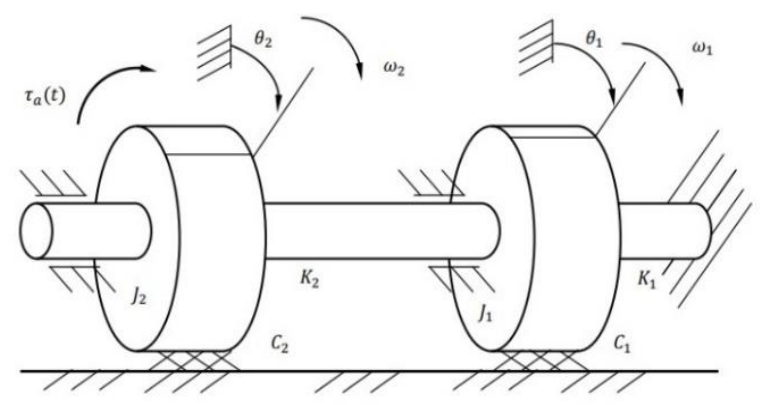

Figure 1. The rotational mechanical plant

The plant comprises of a pole with two rotational plates, the two shafts thought to be adaptable, with solidness constants (K1) and (K2). The two plates, with snapshots of inertia(J1) and(J2), are upheld by orientation whose erosion is immaterial contrasted and the gooey contact components signified by the coefficients $(\mathrm{C} 1)$ and $(\mathrm{C} 2)$. The reference positions for $(\theta 1)$ and $(\theta 2)$ are the positions of the reference marks on the rims of the disks when the system contains no stored energy. The system, shown in Figure 1 is two degrees of freedom system with two inertia elements with independent angular velocities and two shafts with independent angular displacements, so four state variables are required. We have selected $(\theta 1, \theta 2)$ and $(w 1, w 2)$ to be state variables, because they reflect the potential energy stored in each of the shafts and the kinetic energy stored in each disk.
Now from each of the two free body diagrams of disk 1 and 2 , the algebraic sum of torques may be set equal to zero by D'Alembert's law, giving the pair of equations $[17,18]$ :

$$
\begin{aligned}
& J_{1} w_{1}+C_{1} w_{1}+K_{1} \theta_{1}-K_{2}\left(\theta_{2}-\theta_{1}\right)=0 \\
& J_{2} w_{2}+C_{2} w_{2}+K_{2}\left(\theta_{2}-\theta_{1}\right)-\tau_{a}=0
\end{aligned}
$$

Or also, we can re-write the Eq. (1), with two state variables, by:

$$
\begin{aligned}
& \theta_{1}=w_{1} \\
& \theta_{2}=w_{2}
\end{aligned}
$$

So that Eq. (1), becomes:

$$
\begin{aligned}
& J_{1} w_{1}+C_{2} \theta_{1}+K_{1} \theta_{1}-K_{2}\left(\theta_{2}-\theta_{1}\right)=0 \\
& J_{2} w_{1}+C_{2} \theta_{2}+K_{2}\left(\theta_{2}-\theta_{1}\right)-\tau_{a}=0
\end{aligned}
$$

We can find the other two by solving the two equations for $(w 1)^{\circ}$ and $(w 2)^{\circ}$ respectively. Thus,

$$
\begin{aligned}
& w_{1}=\frac{1}{J_{1}}\left[K_{2}\left(\theta_{2}-\theta_{1}\right)-C_{1} \theta_{1}-K_{2} \theta_{2}\right] \\
& w_{2}=\frac{1}{J_{2}}\left[\tau_{a}(t)-C_{2} \theta_{2}-K_{2}\left(\theta_{2}-\theta_{1}\right)\right]
\end{aligned}
$$

To obtain an input-output equation, we rewrite the Eq. (2) in terms of the angular displacements $\left(\theta_{1}\right)$ and $\left(\theta_{2}\right)$ and perform several rearrangements, we can get:

$$
\begin{gathered}
J_{1} \theta_{1}{ }^{*}+C_{1} \theta_{1}+\left(K_{1}+K_{2}\right) \theta_{1}-K_{2} \theta_{2}=0 \\
J_{2} \theta_{2}{ }^{*}+C_{2} \theta_{2} \cdot K_{2}\left(\theta_{2}-\theta_{1}\right)=\tau_{a}(t)
\end{gathered}
$$

From the above-determined conditions and state factors, we see that the given framework is a coupled framework and neither of the over two conditions can't be understood independently. Notwithstanding, from the Eq. (5) We can characterize $\left(\theta_{1}\right)$ as:

$$
\theta_{1}=\frac{1}{K_{2}}\left[\tau_{a}(t)-J_{2} \theta_{2}{ }^{-}-C_{2} \theta_{2}-K_{2} \theta_{2}\right]
$$

Presently by connecting the declaration of $\left(\theta_{1}\right)$ from Eq. (6) into the Eq. (4), we acquire the accompanying fourth order differential equation as far as $\left(\theta_{2}\right)$.

When all is said in done we have to solve higher order differential equations numerically, and afterward we revise them as first order differential equations by presenting state factors. Additionally, so as to have the capacity to understand this fourth order differential condition we would need four starting conditions known or pre-characterized.

By taking the Laplace Transform of each term in the Eqns. (5), (6), under the presumption of zero beginning conditions, and utilizing the linearity property of Laplace Transforms, the exchange capacity can be recorded quickly by assessment of the differential condition. We likewise characterize an exchange work that is yield $\left(\theta_{2}\right)$ output versus input $\left(\tau_{\alpha}\right)$, with the goal that the overall transfer function is: 


$$
\frac{\theta_{2}(s)}{\tau_{a}(s)}=\frac{\left[J_{1} s^{2}+C_{1} s+\left(K_{1}+K_{2}\right)\right]}{J_{1} J_{2} s^{4}+\left(J_{1} C_{2}+J_{2} C_{1}\right) s^{3}+\left(J_{1} K_{2}+J_{2} K_{1}+J_{2} K_{2}+C_{1} C_{2}\right) s^{2}+\left(C_{1} K_{2}+C_{2} K_{1}+C_{2} K_{2}\right) s+K_{1} K_{2}}
$$

We take the following parameters for our numerical simulations as appear in Table 1.

Table 1. Numerical values of the rotational mechanical plant [17]

\begin{tabular}{ccc}
\hline Parameters & Description & Value \\
\hline J1 & Mass moment of inertia for disk 1 & $1.5 \mathrm{Kg} . \mathrm{m}^{2}$ \\
J2 & Mass moment of inertia for disk 2 & $0.5 \mathrm{Kg} \cdot \mathrm{m}^{2}$ \\
K1 & torsional stiffness for disk 1 & $1200 \mathrm{~N} . \mathrm{m} / \mathrm{rad}$ \\
K2 & torsional stiffness for disk 2 & $2000 \mathrm{~N} . \mathrm{m} / \mathrm{rad}$ \\
C1 & frictional damping for disk 1 & $12 \mathrm{~N} . \mathrm{m} . \mathrm{sec}$ \\
$\mathrm{C} 2$ & frictional damping for disk 2 & $22 \mathrm{~N} . \mathrm{m} . \mathrm{sec}$ \\
\hline
\end{tabular}

Substitute these values in Eq. (7), we get:

$$
\frac{\theta_{2}(s)}{\tau_{a}(s)}=\frac{1.5 s^{2}+12 s+3200}{0.75 s^{4}+39 s^{3}+4864 s^{2}+94400 s+2400000}
$$

This is a $4^{\text {th }}$ order system, the poles are the roots of the denominator polynomial. To find this, we use roots ([0.75 39 486494400 2400000]) by MATLAB statement. This gives us roots at:

$$
\begin{aligned}
& -15.8157+70.5617 \mathrm{i} \\
& -15.8157-70.5617 \mathrm{i} \\
& -10.1843+22.5442 \mathrm{i} \\
& -10.1843-22.5442 \mathrm{i}
\end{aligned}
$$

where, the system roots indicate that system is stable, but there is steady-state error can be reduced by using a pre-filter.

\section{MODEL ORDER REDUCTION}

The investigation and amalgamation of higher order frameworks are troublesome and by and large not attractive on financial and computational contemplations [19]. Along these lines, it is important to get a lower arrange framework with the goal that the acquired lower arrange keeps up the attributes of the first framework. These aides in limiting the varieties amid structure and acknowledgment of appropriate control framework parts to be joined to the first system [20].

Display arrange plan is the way toward inferring the lower arrange show from the higher order demonstrate. Demonstrate arrange plan approximates the perplexing framework by basic one. The fundamental point of the detailing is to locate the most ideal estimate of the yield of the first framework. Amid the previous four decades, various amazing assortments of new strategies [21-23] have been produced for getting lower arrange models from higher order straight framework. Every one of these techniques has two points of advantages and disadvantages when attempted on a specific framework.

But in this paper, the Dominant Pole Approximation method (DPA) can be utilized, it is a strategy for approximating a (more complicated) high order framework with a (more straightforward) arrangement of lower arrange if the location of the real part of some of the system poles are adequately near the origin contrasted with the other poles [2427].

Lower arrange (first and second) are well comprehended and simple to describe (speed of framework, motions, damping...,) however it is substantially more troublesome with higher order frameworks. One approach to make numerous such frameworks simpler to consider is to surmise the framework by a lower arrange framework. This guess accepts that the slowest part of the framework dominates the response and that the quicker part(s) of the framework can be disregarded.

To decide predominance, we take the time constants (negative inverse of real part) related to these poles. We see the poles at -15.8157 has time constant $\left(\tau=\frac{1}{15.8157}=0.0632\right)$ where as the other poles have a time constant (The order of magnitude difference tells us that the poles at $(-10.1843$ $+22.5442 \mathrm{i},-10.1843-22.5442 \mathrm{i}$ ) are dominant because they are more near to the imaginary axis. There is no effect on the numerator (zeros).

To reduce the model, we factor out the negligible poles and multiply the dominate poles with each other $(\mathrm{s}+10.1843+22.5442 \mathrm{i})(\mathrm{s}+10.1843-22.5442 \mathrm{i})$, we get the denominator of 2 nd reduced order model:

$$
\frac{\theta_{2}(s)}{\tau_{a}(s)}=\frac{612.57}{s^{2}+20 s+612.57}
$$

After some trial and error change in parameters of transfer function in Eq. (9), we get the proper reduced model as:

$$
\frac{\theta_{2}(s)}{\tau_{a}(s)}=\frac{589}{s^{2}+21.4 s+589}
$$

To represent the way that the frameworks displayed by the original full-arrange exchange work and by the diminished order demonstrate are comparable from a contribution to-yield point of view with zero starting conditions, time-space reactions are appeared as in Figure 2 between original and decreased frameworks. Note that the time reaction plots are indistinguishable for the two models. In spite of the fact that the full-arrange and diminished order exchange capacities give similar contribution to-yield qualities when the underlying conditions are zero, they don't do as such when introductory conditions are incorporated. Above all else, they don't have a similar number of inward state factors, so the quantities of introductory conditions which are required are not the equivalent. The full-arrange demonstrate has 4 states and requires 4 beginning conditions, one for each state variable. The decreased request display has 2 states and requires 2 starting conditions, yet accepting in this work every single introductory condition are zero. The plot demonstrates that the two models meet well. Table 2 indicates transient reaction particulars of the two models. 


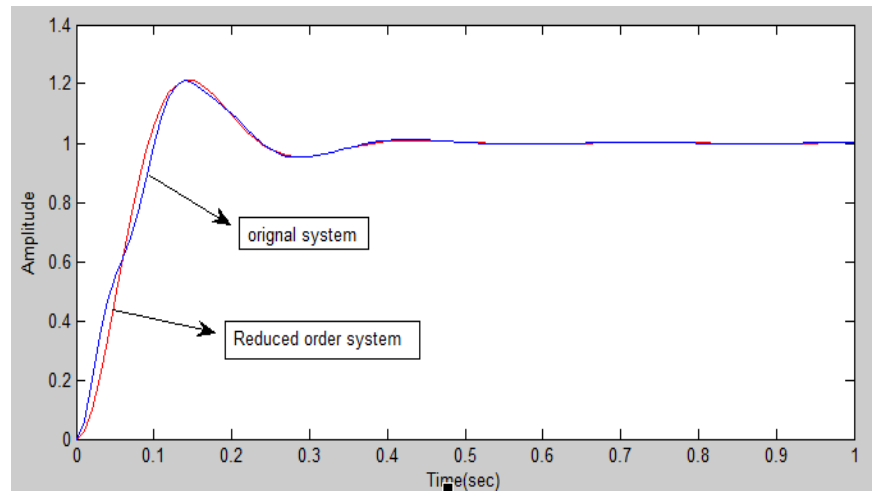

Figure 2. Transient responses comparison between original and reduced models

Table 2. Transient response specifications of both models

\begin{tabular}{cccc}
\hline Model & $\begin{array}{c}\text { Rise- } \\
\text { time- } \\
\text { Tr(sec) }\end{array}$ & $\begin{array}{c}\text { Settling- } \\
\text { time- } \\
\text { Ts(sec) }\end{array}$ & Max.overshoot\% \\
\hline Original & 0.07 & 0.346 & 21.2 \\
Reduced & 0.063 & 0.346 & 21.3 \\
\hline
\end{tabular}

As seen from Table 2 nearly all transient responses specifications are identical. In this paper, order reduction not goal in itself it is a part of design.

\section{CONTROLLERS TYPES}

Their many methods for controlling the rotational mechanical system, as seen, the reduced-order of the system is second order. In this regard, the paper develops different design methods, a PID model-based, full state feedback approach and an intelligent controller technique based on the Genetic Algorithm. The design of controllers, it needs some of the requirements, like, maximum overshot (MP\%), rise( $\mathrm{Tr})$ and settling times(Ts). In this work, and depending on the nature of the system under control (rotational mechanical), the overshoot and vibration avoid from this system, and the reaction is very fast so that the design specifications are:

$$
\begin{gathered}
1-\mathrm{Mp} \%=0 \\
2-\mathrm{Ts}=0.05 \mathrm{sec} .
\end{gathered}
$$

Different control designs are used:

\subsection{PID controller}

(PID) control is the most well-known control calculation utilized in industry and has been all around acknowledged in modern control. The fame of PID controllers can be credited mostly to their vigorous execution in an extensive variety of working conditions and halfway to their practical straightforwardness, this enables architects to work them in a basic, clear way. There are distinctive techniques for tuning (The procedure of setting the ideal increases for $\mathrm{Kp}, \mathrm{Ki}$, and $\mathrm{Kd}$ to get a perfect reaction from a control framework) The Ziegler-Nichols strategy is a famous technique for tuning a PID controller [24]. In this paper, the ideal coefficients dependent on the ITAE criterion for step info can be utilized as pursue:
Let the PID controller as:

$$
D(s)=K_{p}+\frac{K_{i}}{s}+K_{d}
$$

And inset Eq. (11) with the system Eq. (10) and calculate the closed-loop transfer function and equating the denominator of this closed-loop with the optimum coefficients [24]:

$$
\begin{aligned}
& s^{3}+1.7 W_{n} s^{2}+2.15 W_{n}^{2} s+W_{n}^{3} \\
& =s^{3}+\left(21.4+589 K_{d}\right) s^{2}+589 K_{p} s+589 K_{i}
\end{aligned}
$$

Depending on design specifications, the damping ratio is $\varsigma=1$ and from settling time (Ts) the natural damped frequency is $W_{n}=100 \mathrm{rad} / \mathrm{sec}$, so that: $\mathrm{Kp}=363, \mathrm{Ki}=11235, \mathrm{Kd}=0.26$. Figure 3 shows the response of the system with PID controller, where $\mathrm{Ts}=0.092 \mathrm{sec}, \mathrm{Mp}=83.4 \%$ and $\mathrm{Tr}=0.0072 \mathrm{sec}$, as seen the $\mathrm{Mp}$ is very large and need more tuning to reduce it, also in this response, it is necessary to using prefilter to reduce the steadystate error.

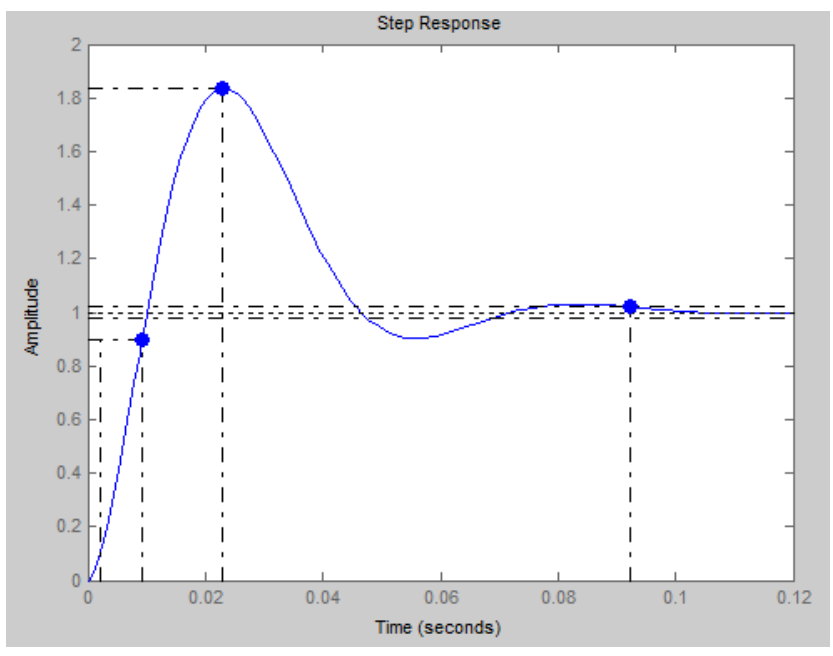

Figure 3. Transient response of the system with PID controller

\subsection{LQR}

LQR technique for controlling the framework, is the statespace approach, can be utilized just for the controllable and recognizable framework and (LQR) is a notable strategy that gives ideally controlled criticism increases to empower closedloop stable and high-performance design of systems [28].

For the deduction of the linear quadratic regulator, we expect the plant to be written in state-space frame $\mathrm{x}=\mathrm{Ax}+\mathrm{Bu}$, and that the majority of then states $\mathrm{x}$ are accessible for the controller. The input gain is a grid $\mathrm{K}$, actualized as:

$\mathrm{u}=-\mathrm{K}(\mathrm{x}-\mathrm{xdesired})$. The framework elements are then composed as:

$$
\dot{\mathrm{x}}=(\mathrm{A}-\mathrm{BK}) \mathrm{x}+\mathrm{BKxdesired})
$$

Pole-placement is the way toward putting the shafts of (A$\mathrm{BK})$ in stable, reasonably damped areas in the unpredictable plane.

The principal thought in LQR control configuration is to limit the quadratic cost capacity of: 


$$
J=\int_{0}^{\infty}\left(x^{T} Q x+u^{T} R u\right) d t
$$

where, Q and R are the positive-distinct Hermitian or genuine symmetric framework. Note that the second term on the correct side record for the use of the vitality on the control endeavors. The matrix $\mathrm{Q}$ and $\mathrm{R}$ decide the overall significance of the error and the use of this vitality. The LQR configuration chooses the weight lattice $\mathrm{Q}$ and $\mathrm{R}$ with the end goal that the exhibitions of the closed-loop framework can fulfill the ideal prerequisites referenced before. The determination of $\mathrm{Q}$ and $\mathrm{R}$ is pitifully associated with the execution specifications, and a specific measure of experimentation is required with a computer simulation before a palatable plan result.

For reasons unknown, paying little respect to the estimations of $\mathrm{Q}$ and $\mathrm{R}$, the cost work has a one of a kind least that can be acquired by illuminating the Algebraic Riccati Equation. The parameters $\mathrm{Q}$ and $\mathrm{R}$ can be utilized as plan parameters to punish the state factors and the control signals. The bigger these qualities are, the more you punish these signs. Fundamentally, picking an extensive incentive for R implies you attempt to balance out the framework with less (weighted) vitality. This is generally called a costly control procedure. Then again, picking a little an incentive for $\mathrm{R}$ implies you would prefer not to punish the control flag (shoddy control system). So also, in the event that you pick an extensive incentive for Q implies you endeavor to settle the framework with the minimum conceivable changes in the states and expansive Q suggests less worry about the adjustments in the states. Since there is an exchange off between the two, you might need to keep Q as I (personality framework) and just change R. You can pick an extensive R, if there is a limit on the control yield motion (for example, if huge control signals present sensor commotion or cause actuator's immersion), and pick a little $\mathrm{R}$ if having an expansive control flag isn't an issue for your system [29].

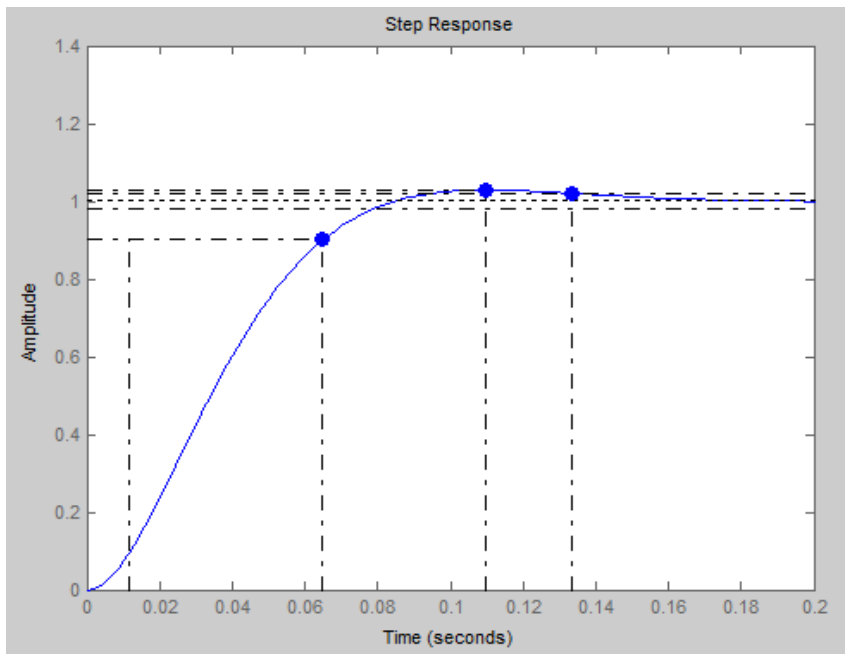

Figure 4. Transient response of the system with LQR controller

On the off chance that you realize the ideal closed-loop pole areas, you can utilize the MATLAB directions place or acker. Another alternative is to utilize the lqr order which restores the ideal controller increase accepting a linear plant, quadratic cost capacity, and reference equivalent to zero.

Since the desired closed loop poles are:

$$
\begin{aligned}
& S_{1,2}=-\varsigma W_{n} \pm j W_{n} \sqrt{1-\varsigma^{2}} \\
& S_{1,2}=-100 \pm 0 j
\end{aligned}
$$

The optimum choosing of $(\mathrm{Q})$ as [28]:

$\mathrm{Q}=\mathrm{C}^{\prime *} \mathrm{C}$ and let $\mathrm{R}=0.1$.

Using lqr Matlab statement, the desired gains $\mathrm{K}=\left[\begin{array}{ll}\mathrm{K} 1 \mathrm{~K} 2\end{array}\right]$, can be found:

$$
\mathrm{K}=[1862.643 .3]
$$

Figure 4 shows the transient response of the system with LQR controller. As seen the (Ts) is very large and need more tuning to reduce it, also in this response, it is necessary to using prefilter with gain (3.163) to reduce the steady-state error.

\subsection{Optimal control design}

Intelligent control alludes to ways to deal with control frameworks configuration, demonstrating, distinguishing proof, and task that utilization computerized reasoning methods, for example, fuzzy logic, neural systems, machine learning, evolutionary calculation, and genetic algorithms [30]. In this paper the usage of the (PID) controller with genetic algorithm (G.A) tuning.

G.A is versatile heuristic pursuit calculation dependent on the developmental thoughts of regular determination and hereditary qualities. In that capacity, they speak to a keen misuse of an irregular pursuit used to unravel enhancement problems [31].

It is as often as possible used to discover ideal or close ideal answers for troublesome issues which generally would take a lifetime to unravel. It is much of the time used to tackle enhancement issues, in research, and in machine learning. Advancement alludes to finding the estimations of contributions to such a way, to the point that we get the "best" yield esteems. The meaning of "best" fluctuates from issue to issue, yet in scientific terms, it alludes to amplifying or limiting at least one target capacities, by shifting the information parameters [32].

G.A can convey a "sufficient" arrangement "quick enough". This makes (G.A) appealing for use in taking care of advancement issues.

The genetic algorithm utilizes four fundamental advances:

Stage 1 . Instate the parameter with a populace of arbitrary arrangements, for example, hybrid rate, change rate, number of groups, and number of ages. Decide the coding mode.

Stage 2. Figure and assess the estimation of wellness work.

Stage 3 . Continue with hybrid and change activity and make up the new group.

Stage 4. Repeat Step 2, until the best esteem is gotten. Figure 5 shows the flow-diagram of G.A.

In this paper, toolbox optimization of MATLAB (R2012a) with the accompanying administrators is executed for the ideal structure of the G.A-PID controller.

(G.A) has additionally been generally used to tune the parameters of PID. It is outlined as pursues:

GA at first produces an arbitrary populace, which is executed with little populace estimate so as to enable the controller to be improved and combine at a quicker rate. The underlying populace is set by encoding the PID parameters, and into parallel strings known as a chromosome. The wellness of every chromosome is determined by changing 
over its parallel string into genuine esteem which speaks to the PID parameters. Each arrangement of PID parameters is passed to the PID controller. The entire reaction of the framework for every PID parameter esteem and its underlying wellness esteem is registered utilizing performance index like [33]:

$$
I S E=\int_{0}^{\infty}[e(t)]^{2} d t
$$

where, (ISE) is Integral-Square-Error.

We define the fitness of the chromosomes as:

$$
\text { fitnessvalue }=\frac{1}{\text { performanceindex }(I S E)}
$$

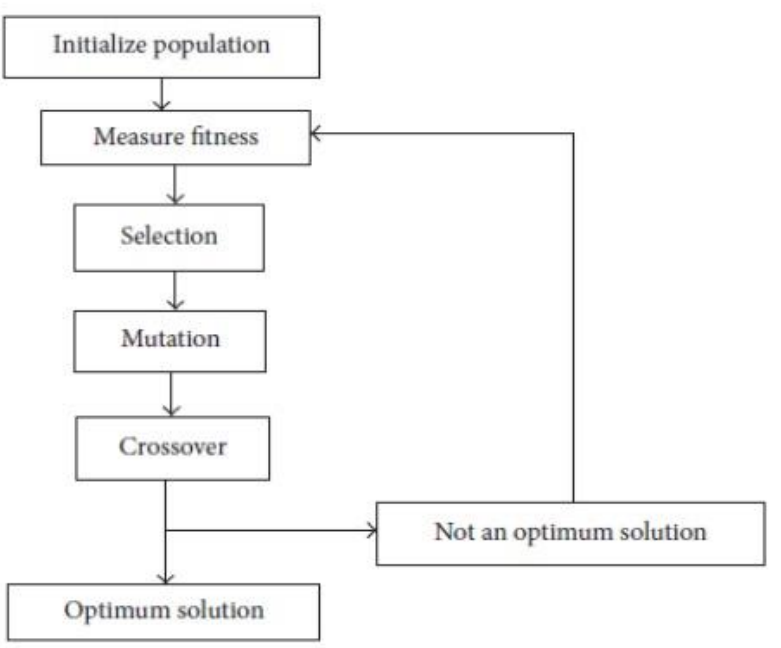

Figure 5. Flow-diagram of G.A

A weighted mix of these three cost capacities. This procedure will experience Steps 2 and 3 until the finish of the ages where the best wellness esteem is accomplished. A definitive point of GA is to look for worldwide PID esteems $(\mathrm{Kp}, \mathrm{Ki}, \mathrm{Kd})$ with least wellness incentive to work the rotational Mechanical System in the whole range. The subtleties of GA parameters utilized in the recreation have appeared in Table 3, which can assess the ideal PID increases dependent on the controlled frameworks.

Table 3. GA parameters

\begin{tabular}{cc}
\hline Parameter & Value \\
\hline Population size & 50 \\
Stopping criteria & Maximum generation 100 \\
Selection method & Roulette wheel \\
Crossover function & Intermediate \\
Mutation function & Uniform \\
\hline
\end{tabular}

By substituting the qualities in the genetic algorithm we get the estimation of $\mathrm{Kp}, \mathrm{Ki}$ and $\mathrm{Kd}$ more than 100 generation as appeared:

$$
\mathrm{Kp}=497.411, \mathrm{Ki}=48973.124, \mathrm{Kd}=15.191 .
$$

Figure 6 shows the transient response of the system with G.A-PID controller.

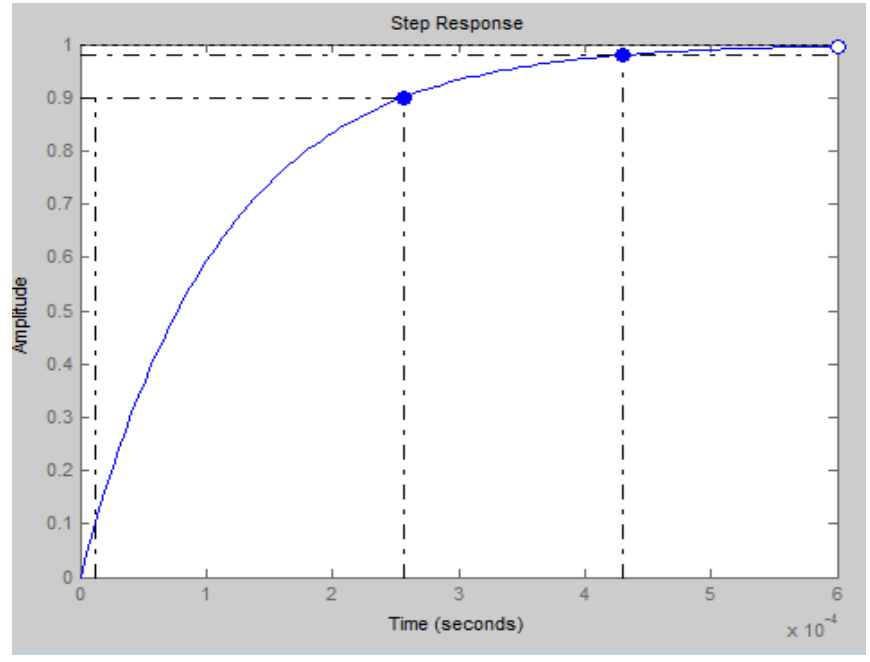

Figure 6. Transient response of the system with G.A-PID controller

where, $\mathrm{Mp}=0 \%$, Ts $=0.000431 \mathrm{sec}$ and $\mathrm{Tr}=0.000245 \mathrm{sec}$. Here all the design requirements are satisfied. Table 4 shows the comparison of the results obtained from the step response of the system. The parameters considered are the Maximum Overshoot, Rise time, Settling time \& steady state error. Better results are obtained using GA compared with classically tuned parameters, as seen from table, the disadvantages of PID and LQR are large overshot and response speed is sluggish due to large settling and rise time, when compared with GA-PID. The method of LQR needs prefilter to compensate steady-state error and this added extra cost.

Table 4. Comparison between all controllers

\begin{tabular}{ccccc}
\hline $\begin{array}{c}\text { Controller } \\
\text { types }\end{array}$ & $\begin{array}{c}\text { Settling } \\
\text { time(sec) }\end{array}$ & $\begin{array}{c}\text { Rise } \\
\text { time(sec) }\end{array}$ & Max.overshot\% & $\begin{array}{c}\text { Steady- } \\
\text { state } \\
\text { error }\end{array}$ \\
\hline PID & 0.092 & 0.0072 & 83.4 & $\begin{array}{c}0 \\
\text { LQR }\end{array}$ \\
0.133 & 0.053 & 2.86 & $\begin{array}{c}\text { (need } \\
\text { prefilter) } \\
0\end{array}$ \\
G.A-PID & 0.00043 & 0.00024 & 0 & 0 \\
\hline
\end{tabular}

\section{CONCLUSIONS}

This paper exhibits an advancement strategy for PID control parameters for the Reduced-Order of Rotational Mechanical System by GA as an inquiry method with least data explicit to the framework, for example, the defined fitness function. From the outcomes, it is exhibited that the streamlined PID enhance the exhibitions of the Rotational Mechanical System so as to accomplish least settling time with no overshoot and zero steady state error. The complementary of ITAE foundation is changed to be a proper wellness work for GA to assess the control execution of the given input gains. Tuning through genetic algorithms prompted attractive closed-loop step reaction. Results contrasted positively with those acquired through the traditional Ziegler - Nichols' tuning strategy. As some of the time is the situation with this strategy, there was no requirement for further manual changes in accordance with the PID picks up when programmed tuning was utilized. The programmed technique was even fit for giving increase esteems where the established strategy couldn't be applied. (LQR) is utilized additionally as a strategy for correlation. The organized PID with the Genetic Algorithm has significantly 
snappier response than the setup system (PID) and (LQR). These refinements appear the extent that the rise time and the settling time. Also, this works shows, the reduced model (2nd order), instead of the original model (4th order), gives good behavior and satisfied all transient response requirements, and reduce the complexity of mathematical analysis and design steps. The structure of the control frameworks by utilizing GAs can help the architect in working with a diminished number of plan techniques to build up the kind of the controller and probability of effortlessly designing the dynamic behavior of the control framework. Finally many issues future work for model reduction can be used, such optimization methods or MATLAB statement options and for controller using artificial methods, such sliding mode or neural network and fuzzy logic.

\section{ACKNOWLEDGMENT}

The author would like to thank Mustansiriyah University (www.uomustansiriyah.edu.iq) Baghdad - Iraq for its support in the present work.

\section{REFERENCES}

[1] Antoulas, A.C. (2005). Approximation of large-scale dynamical systems. Approximation of Large-Scale Dynamical Systems. https://doi.org/10.1137/1.9780898718713

[2] Nandong, J., Seer, Q.H. (2017). Stabilising PID tuning for a class of fourth-order integrating no minimum-phase systems. International Journal of Control, 92(6): 12261242. https://doi.org/10.1080/00207179.2017.1387289

[3] Obinata, G., Anderson, B. (2001). Model Reduction for Control System Design. Springer, Vienna, Austria.

[4] Soni, Y.K., Bhatt R. (2003). PSO optimized reduced order PID controller design. International Journal of Advanced Research in Computer Engineering \& Technology, 2(3): 989-992.

[5] Shamash, Y. (1975). Model reduction using the Routh stability criterion and the pole ap-proximation technique. International Journal of Control, 21(3): 475-484. https://doi.org/10.1080/00207177508922004

[6] Astr“om, K.J., Murray, R.M. (2008). Feedback Systems an Introduction for Scientists and Engineers. Princeton University Press.

[7] Jones, R.W., Tham M.T. (2007). PID design approaches for higher-order plants. IEEE, 2007 European Control Conference.

[8] Palaniswami, S., Sivanandam, S.N. (2000). Design of PID controller using lower order model. National Symposium on Intelligent Measurement and Control, NSMIC-2000, MIT, Chennai, India, pp. 290-296.

[9] Gildin, E. (2006). Development of low-order controllers for high-order reservoir models and smart wells. SPE Annual Technical Conference and Exhibition, San Antonio, Texas, USA. https://doi.org/10.2118/102214MS

[10] Rajani, S.H., Nair, U. (2013). Performance comparison of H-infinity and LQR controllers for the pressure regulation of a hypersonic wind tunnel. International Journal of Modern Engineering Research, 3(3): 16231629.

[11] Vaishnav, S.R., Khan, Z.J. (2007). Design and performance of PID and fuzzy logic controller with smaller rules set for higher order system. Proceedings of the World Congress on Engineering and Computer Science 2007, WCECS 2007, San Francisco, USA.

[12] Varsek, A., Urbacic, T., Filipic, B. (1993). Genetic algorithms in controller design and tuning. IEEE Transactions on Systems, Man, and Cybernetics, 23(5): 1330-1339. https://doi.org/10.1109/21.260663

[13] Gaing, Z.L. (2004). A particle swarm optimization approach for optimum design of PID controller in AVR system. IEEE Transaction on Energy Conversion, 19(2): 384-391. https://doi.org/10.1109/TEC.2003.821821

[14] Zhao, J., Li, T., Qian, J. (2005). Application of particle swarm optimization algorithm on robust PID controller tuning. Advances in Natural Computation - Springer, 948-957. https://doi.org/10.1007/11539902_118

[15] Roland, S.B. (2001). Advanced Control Engineering. Butterworth-Heinemann.

[16] Civelek, C. (2006). Mathematical modelling of rotational mechanical elements of higher order and their characteristics. Mathematical and Computer Modelling, 43(7-8): 957-964. https://doi.org/10.1016/j.mcm.2005.09.023

[17] Close, C.M., Frederick, D.K., Newell, J.C. (2002). Modeling and Analysis of Dynamic Systems. 3rd edition, John Wiley and Sons Inc.

[18] Kluever, C.A. (2015). Dynamics Systems: Modeling, Simulation and Control. Wiley-Publication.

[19] Kumari, S., Taneja, R. (2015). Model order reduction: An approach towards a simpler system design. International Journal for Research in Applied Science \& Engineering Technology (IJRASET), 3(V).

[20] Singh, V.P., Chandra, D. (2010). Routh approximation based model reduction using series expansion of interval systems. IEEE International Conference on Power, Control \& Embedded Systems (ICPCES), Allahabad, India. https://doi.org/10.1109/ICPCES.2010.5698658

[21] Deepa, S.N., Sugumaran, G. (2011). Design of PID controller for higher order continuous systems using MPSO based model formulation technique. International Journal of Electrical and Information Engineering, 5(8): 949-955.

[22] Chowdhury, A., Das, S. (2013). Analysis and design of missile two loop autopilot. Advance in Electronic and Electric Engineering, 3(8): 959-964.

[23] Mukherjee, S., Satakshi, Mittal, R.C. (2005). Model order reduction using response-matching technique. Journal of the Franklin Institute, 342(5): 503-519. https://doi.org/10.1016/j.jfranklin.2005.01.008

[24] Anand, D.K, Zmood, R.B. (1995). Introduction to Control Systems. $3^{\text {rd }}$ Edition, Butterworth Heinemann Ltd.

[25] Martins, N., Lima, L.T.G., Pinto, H.J.C.P. (1996). Computing dominant poles of power system transfer functions. Power Systems. IEEE Transactions, 11(1): 162-170. https://doi.org/10.1109/59.486093

[26] Rommes, J., Martins, N. (2006) Efficient computation of transfer function dominant poles using subspace acceleration. IEEE Transactions on Power Systems, 21(3):

$1218-1226$ https://doi.org/10.1109/TPWRS.2006.876671

[27] Wilhelmus, H., Henk, A., Joost, R. (2008). Model Order Reduction: Theory, Research Aspects and Applications. Springer, Berlin, Heidelberg. 
[28] Louis, W. (1995). Handbook of control systems Engineering. John-Wiley Inc.

[29] Siradjuddin, I., Setiawan, B., Fahmi, A. (2017). State space control using LQR method for a cart-inverted pendulum linearised model. International Journal of Mechanical and Mechatronics Engineering, 17(1): 119126.

[30] Hiwarkar, T.A., Iyer, R.S. (2013). New applications of soft computing, artificial intelligence, fuzzy logic \& genetic algorithm in bioinformatics. International Journal of Computer Science and Mobile Computing, 2(5): 202-207.

[31] Davis (1990). Handbook of Genetic Algorithms, VNR Comp. Library.

[32] Kumar, S.M.G., Jain, R., Anantharaman, N., Dharmalingam, V., Begum, K.M.M.S. (2008). Genetic algorithm based PID controller tuning for a model bioreactor. Indian Chemical Engineer, Indian Institute of Chemical Engineers, 50(3): 214-226.

[33] Tandon, B., Kaur, R. (2011). Genetic algorithm based parameter tuning of PID controller for composition control system. International Journal of Engineering

Science and Technology, 3(8): 6705-6711.

\section{NOMENCLATURE}

$\mathrm{J}$

$\mathrm{K}$

$\mathrm{C}$

$\theta$

W

Ta

DPA

GA

PID

LQR

ISE

$\mathrm{Wn}$

$\mathrm{Mp}$

Ts

$\operatorname{Tr}$

$\zeta$
Mass moment of inertia for disk, Kg.m²

Torsional stiffness for disk, N.m/rad

Frictional damping for disk, N.m.sec

Angular displacement, rad

Angular velocity, $\mathrm{rad} / \mathrm{sec}$

Input torque, N.m

Dominant Pole Approximation

Genetic algorithm

Proportional integral derivative

Linear-Quadratic Regulator

ntegral square error

Natural damping frequency, $\mathrm{rad} / \mathrm{sec}$

Maximum overshot

Setling time,sec

Rise time,sec

Damping ratio 